TRANSACTIONS OF THE

AMERICAN MATHEMATICAL SOCIETY

Volume 352, Number 10, Pages 4815-4841

S 0002-9947(00)02567-8

Article electronically published on June 14, 2000

\title{
LOCAL STRUCTURE OF SCHELTER-PROCESI SMOOTH ORDERS
}

\author{
LIEVEN LE BRUYN
}

\begin{abstract}
In this paper we give the étale local classification of SchelterProcesi smooth orders in central simple algebras. In particular, we prove that if $\Delta$ is a central simple $K$-algebra of dimension $n^{2}$, where $K$ is a field of trancendence degree $d$, then there are only finitely many étale local classes of smooth orders in $\Delta$. This result is a non-commutative generalization of the fact that a smooth variety is analytically a manifold, and so has only one type of étale local behaviour.
\end{abstract}

\section{INTRODUCTION}

The quest for a suitable notion of a smooth non-commutative variety is still wide open. Up till now most attention has been focussed on algebras having excellent homological properties. However, even in the case when these algebras are finite modules over their centers, a local characterization of regular algebras seems to be out of reach at the moment.

Still, in the case of algebras finite over their centers we have a workable alternative. In [10] W. Schelter introduced and studied smooth algebras by requiring that they have a formal smoothness lifting property with respect to test-objects satisfying the same (or smaller-size) polynomial identities.

Later, C. Procesi gave a slight variation of this definition by restricting to algebras having a trace map satisfying the $n$-th formal Cayley-Hamilton identity, [8]. He proved that these smooth non-commutative algebras have a nice geometrical characterization.

Consider the affine variety $X_{A}$ of all $n$-dimensional representations of $A$ (compatible with the trace maps). Then $A$ is smooth if and only if $X_{A}$ is a smooth (commutative) variety.

In case $A$ is a smooth order in a central simple algebra, the quotient variety of $X_{A}$ under the natural action of $P G L_{n}$ is the variety corresponding to the center $Z$ of $A$. That is, to a maximal ideal $m$ of $Z$ corresponds a closed orbit in $X_{A}$ determining a semi-simple $n$-dimensional representation $M_{m}$ of $A$ with decomposition in simple components, say

$$
M_{m}=S_{1}^{\oplus e_{1}} \oplus \ldots \oplus S_{r}^{\oplus e_{r}}
$$

Received by the editors July 10, 1997.

2000 Mathematics Subject Classification. Primary 16R30.

The author is a research director of the NFWO. 
where the $S_{i}$ are distinct simple $A$-modules of dimension $d_{i}$ occurring with multiplicity $e_{i}$. We then say that $m$ has representation type $\tau=\left(e_{1}, d_{1} ; \ldots ; e_{r}, d_{r}\right)$ with $\sum e_{i} d_{i}=n$.

We want to determine the étale local structure of $A$ near $m$, that is, we want to determine the algebra structure of

$$
A_{m}^{s h}=A \otimes_{Z} Z_{m}^{s h},
$$

where $Z_{m}^{s h}$ is the strict henselization of $Z$ at $m$. Alternatively, we want to determine the $m$-adic completion of $A$.

We succeed in doing this by applying the Luna slice theorems at a point $x$ of the smooth variety $X_{A}$ lying in the closed orbit corresponding to $m$. The stabilizer group in $x$ is, with notations as above, $G L(\mathbf{e})=G L_{e_{1}} \times \ldots \times G L_{e_{r}}$ embedded in $G L_{n}$ using the numbers $d_{i}$. The étale local structure of $A$ and $Z$ near $m$ is fully determined by the $G L(\mathbf{e})$-module structure of the normal space $N_{x}$ to the orbit of $x$.

The isotypical decomposition of $N_{x}$ as $G L(\mathbf{e})$-module can be encoded in a 'local chart' $C=(M, \mathbf{e})$, where the map $M$ is a labeled directed marked graph on $r$ vertices and $N_{x}$ is the space of representations $R(M, \mathbf{e})$ of $M$ of dimension vector $\mathbf{e}=\left(e_{1}, \ldots, e_{r}\right)$.

If $A$ is a smooth order in a central simple algebra $\Delta$ of dimension $n^{2}$ over its center $K$ which is a field of trancendence degree $d$, then $C=(M, \mathbf{e})$ must be such that the dimension of the quotient variety $R(M, \mathbf{e}) / G L(\mathbf{e})$ is equal to $d$ and there are simple e-dimensional representations of $M$. Both conditions can be expressed in terms of the Euler-form of the map $M$.

We give a method to enumerate all charts that can occur for a given dimension $d$. Moreover, these local charts not only determine the étale local structure of $Z$ and $A$ in $m$ but also contain enough information to determine the local charts at nearby points (and hence the local structure).

To illustrate our general results, let us give the étale local structure of smooth orders in dimension $d=2$.

In this case the only local charts $C=(M, \mathbf{e})$ that can occur have as underlying map $M$ a directed graph of the form

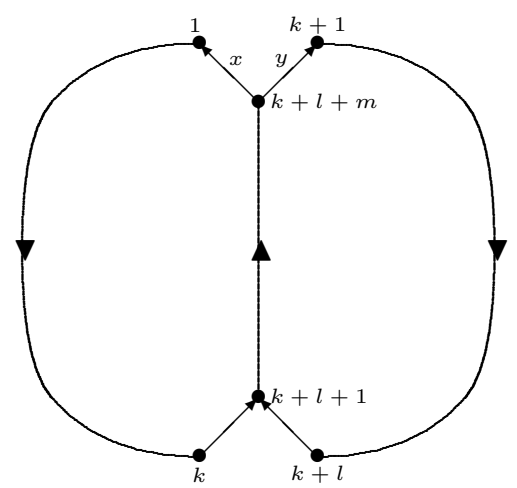

$A_{k l m}$

and dimension vector $\mathbf{e}=(1, \ldots, 1)$. This means that $A$ can only have semi-simple $n$-dimensional representations of type $\tau=\left(1, d_{1} ; \ldots ; 1, d_{k+l+m}\right)$ with $\sum d_{i}=n$. 
From the local chart we can determine the local structure of $Z$ and $A$ in $m$.

Theorem 1.1. With notations as before, we have that $\hat{Z}_{m} \simeq \mathbb{C}[[x, y]]$ and

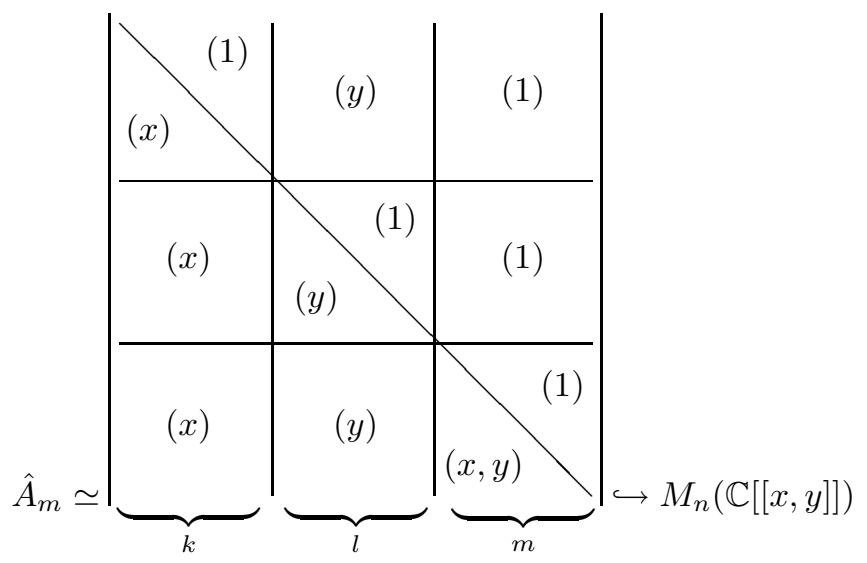

where at place $(i, j)$ (for every $1 \leq i, j \leq k+l+m$ ) there is a block of dimension $d_{i} \times d_{j}$ with entries the indicated ideal of $\mathbb{C}[[x, y]]$.

In particular, we see that a smooth two-dimensional order has a smooth center, its non-Azumaya locus has normal crossings as its worst singularities, and $A$ is étale locally split at every $m$.

In higher dimensions $d$ it is no longer true that the center of a smooth order is smooth nor that a smooth order is étale locally split everywhere.

\section{Smooth NON-COMmutative ALGEBRAS}

In this section we recall the definition of smooth algebras satisfying polynomial identities due to W. Schelter [10] as modified by C. Procesi 8], and draw some consequences.

Throughout, we work over an algebraically closed field of characteristic zero, which we will denote by $\mathbb{C}$. Recall that a commutative affine algebra $A$ is regular if and only if $A$ satisfies the formally smooth lifting property, see for example [3].

A test-object $(C, N)$ is a commutative algebra $C$ together with an ideal $N$ satisfying $N^{k}=0$. The lifting property requires that every diagram

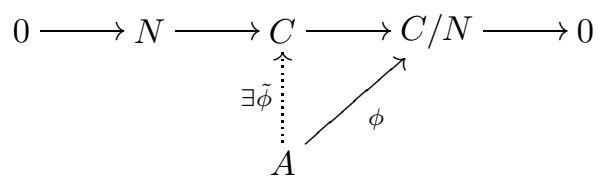

with $\phi$ an algebra morphism can be completed.

If $A$ is non-commutative, one can define $A$ to be smooth provided $A$ has the above lifting property for test-objects $(C, N)$ with $C$ restricted to some suitable category of not necessarely commutative algebras.

A first idea (as pursued for example by J. Cuntz and D. Quillen [1, 2]) is to vary $C$ in the category of all associative algebras. This is too stringent a condition, as it is equivalent to $\Omega_{A}$ being a projective $A^{e}=A \otimes_{\mathbb{C}} A^{o p}$-module, where $\Omega_{A}$ is the kernel of the multiplication map $\mu: A^{e} \rightarrow A$, see [10, lemma 2.3]. Therefore, if $A$ is a regular commutative algebra, it can only be 'smooth' in this sense provided $\operatorname{Kdim} A \leq 1$. 
A more realistic approach is to restrict the class of test-objects $C$ to those algebras having similar commutativity conditions as $A$. Schelter defines a smooth algebra by requiring that if $A$ satisfies the identities of $n \times n$ matrices, then we consider only test-objects $C$ of pi-degree $\leq n$. In this way we recover the commutative characterization of regular algebras as the $n=1$ case.

Later, Procesi [8] gave a slightly different definition of smoothness which allows a geometric study of these algebras. He considers only algebras $A$ having a linear trace map $\operatorname{tr}: A \rightarrow A$ satisfying the following conditions for all $a, b \in A$ :

1. $\operatorname{tr}(a b)=\operatorname{tr}(b a)$,

2. $\operatorname{tr}(a) b=b \operatorname{tr}(a)$,

3. $\operatorname{tr}(\operatorname{tr}(a) b)=\operatorname{tr}(a) \operatorname{tr}(b)$.

In particular, the image of $t r$ is a subalgebra of the center of $A$. We can then define the $n$-th Cayley-Hamilton polynomial formally. In $\mathbb{Q}\left[x_{1}, \ldots, x_{n}\right]$ one defines the elementary symmetric functions by the identity

$$
\prod\left(t-x_{i}\right)=\sum_{i=0}^{n}(-1)^{i} \sigma_{i} t^{d-i}
$$

and the power sums functions $\tau_{k}=\sum x_{i}^{k}$. Because $\left\{\sigma_{i}\right\}$ and $\left\{\tau_{i}\right\}$ are generators of the symmetric functions, there are functions with rational coefficients such that

$$
\sigma_{k}=p_{k}\left(\tau_{1}, \ldots, \tau_{n}\right),
$$

and we define the functions $\sigma_{k}$ on $A$ formally as

$$
\sigma_{k}(a)=p_{k}\left(\operatorname{tr}(a), \operatorname{tr}\left(a^{2}\right), \ldots, \operatorname{tr}\left(a^{n}\right)\right)
$$

and the $n$-th Cayley-Hamilton polynomial for $A$ as

$$
\chi_{n, a}(t)=\sum_{i=0}^{n}(-1)^{i} \sigma_{i}(a) t^{n-i} .
$$

Definition 2.1. We say that an algebra $A$ with a trace function $t r$ is an $n$-th Cayley-Hamilton algebra if

1. for all $a \in A$ we have $\chi_{n, a}(a)=0$ in $A$, and

2. $\operatorname{tr}(1)=n$.

By $C H_{n}$ we will denote the category with objects $\left(A, t r_{A}\right)$ algebras $A$ with a trace function $\operatorname{tr}_{A}$ which are $n$-th Cayley-Hamilton algebras, and morphisms

$$
f:\left(A, t r_{A}\right) \rightarrow\left(B, t r_{B}\right)
$$

which are algebra morphisms which are trace preserving, that is, the diagram below is commutative:

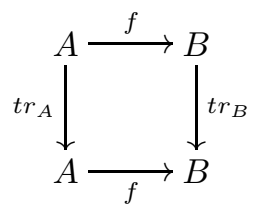

The archetypical example of an $n$-th Cayley-Hamilton algebra is an order in a central simple algebra. 
Example 2.2. Let $K$ be the function field of a normal variety $X$ and let $\Delta$ be a central simple $K$-algebra of dimension $n^{2}$ over $K$. By the classical theory of Brauer groups we know that

$$
\Delta \otimes_{K} L \simeq M_{n}(L)
$$

for some finite Galois extension $L$ of $K$ with Galois group $G$.

On $M_{n}(L)$ we have the usual trace map $\operatorname{Tr}$, and for $\delta \in \Delta$ we can define $\operatorname{tr}(\delta)=$ $\operatorname{Tr}(\delta \otimes 1)$. As the image is invariant under the Galois group, it follows that $\operatorname{tr}(\delta) \in$ $K$, and it is called the reduced trace map on $\Delta$.

Let $R$ be the coordinate ring of an affine open set of $X$ and let $A$ be an $R$-order in $\Delta$. That is, $A$ is a finitely generated $R$-submodule of $\Delta$ such that $A . K=\Delta$. As $R$ is integrally closed, it follows that $\operatorname{tr}(a) \in R$ for all $a \in A$; and as we are in characteristic zero, $\operatorname{tr}(A)=R$. Clearly, $A$ equipped with the reduced trace is an $n$-th Cayley-Hamilton algebra.

One can study $n$-th Cayley-Hamilton algebras via (commutative) algebraic geometry and geometric invariant theory.

Let $\left(A, \operatorname{tr}_{A}\right) \in C H_{n}$ be $m$-generated, that is, there are elements $a_{1}, \ldots, a_{m} \in A$ such that the subalgebra in $C H_{n}$ generated by them is equal to $A$ (note that this is weaker than $A$ being generated as algebra by $m$ elements). Consider

$$
X_{A}=\left\{\phi:\left(A, t r_{A}\right) \rightarrow\left(M_{n}(\mathbb{C}), T r\right) \text { in } C H_{n}\right\} .
$$

By taking the images $\phi\left(a_{i}\right) \in M_{n}(\mathbb{C})$ for $1 \leq i \leq m$ it is clear that $X_{A}$ is a closed subvariety of the affine space $M_{n}(\mathbb{C})^{\oplus m}$. There is a natural action of $P G L_{n}$ on $M_{n}(\mathbb{C})^{\oplus m}$ by simultanous conjugation. Clearly, $X_{A}$ is a $P G L_{n}$-stable closed subvariety of $M_{n}(\mathbb{C})^{\oplus m}$. If we denote by $C H_{n}^{(m)}$ the subcategory of $C H_{n}$ consisting of algebras which are trace generated by $m$ elements, we have

Theorem 2.3 (Procesi, [8]). The functor

$$
C H_{n}^{(m)} \rightarrow P G L_{n} \text {-closed subvarieties of } M_{n}(\mathbb{C})^{\oplus m}
$$

assigning $X_{A}$ to $A \in C H_{n}^{(m)}$ has a left inverse. This inverse assigns to a $P G L_{n}$ closed subvariety $X$ the ring of $P G L_{n}$-equivariant maps $X \rightarrow M_{n}(\mathbb{C})$, or equivalently, the ring of concomitants

$$
M_{n}(\mathbb{C}[X])^{P G L_{n}} .
$$

This means that we can recover $A \in C H_{n}^{(m)}$ from the affine $P G L_{n}$-variety $X_{A}$ as $A \simeq M_{n}\left(\mathbb{C}\left[X_{A}\right]\right)^{P G L_{n}}$. The embedding $j_{A}: A \hookrightarrow M_{n}\left(\mathbb{C}\left[X_{A}\right]\right)$ has the following universal property. Let $C$ be a commutative algebra and $F: A \rightarrow M_{n}(C)$ a morphism in $C H_{n}$ (with the usual trace map on $M_{n}(C)$ ). Then there is a uniquely determined morphism $f: \mathbb{C}\left[X_{A}\right] \rightarrow C$ making the diagram below commutative:

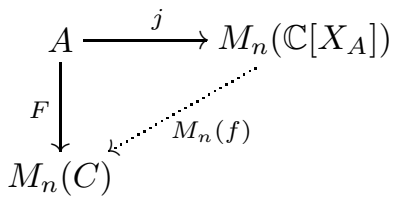

Definition 2.4. An affine algebra $\left(A, t r_{A}\right)$ in $C H_{n}$ is said to be smooth if and only if for every test-object $(C, N)$, where $\left(C, t r_{C}\right) \in C H_{n}, N$ a nilpotent ideal (invariant under the trace map and such that also $\left.\left(C / N, \overline{t r_{C}}\right) \in C H_{n}\right)$ and every 
morphism $\phi:\left(A, \operatorname{tr}_{A}\right) \rightarrow\left(C / N, \overline{\operatorname{tr}_{C}}\right)$ in $C H_{n}$, the diagram below can be completed in $\mathrm{CH}_{n}$ :

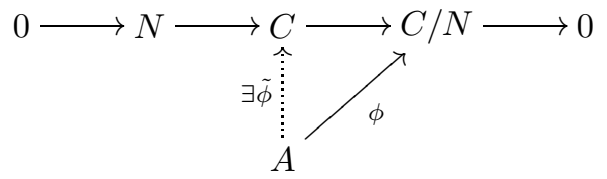

Procesi gave the following geometric characterization of smooth algebras.

Proposition 2.5 (Procesi [8]). The following assertions are equivalent:

1. $A$ is a smooth algebra in $\mathrm{CH}_{n}$.

2. $X_{A}$ is a smooth (commutative) variety.

Proof. (1) $\Rightarrow(2)$. Let $(C, N)$ be a commutative test-object for $\mathbb{C}\left[X_{A}\right]$. We have to lift the map $\mathbb{C}\left[X_{A}\right] \rightarrow C / N$ to $C$. By smoothness in $C H_{n}$ of $A$ we can complete with $F$ the diagram

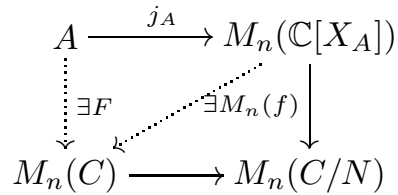

but then by the universal property of $j_{A}$ there is a uniquely determined map $f$ : $\mathbb{C}\left[X_{A}\right] \rightarrow C$ which is the required lift.

The converse implication makes essential use of the Reynolds operator in invariant theory, see [8].

From now on we will restrict attention to prime smooth algebras $A$, or equivalently, to the case when $X_{A}$ is an irreducible smooth variety. In this case we can show that $A$ has to be an order over a normal domain. In fact, we have the following geometric interpretation of the center $Z$ of $A$.

Lemma 2.6. The center $Z$ of $A$ is the coordinate ring of the quotient variety $X_{A} / P G L_{n}$, and is a Cohen-Macaulay normal domain.

Proof. By the characterization of smooth algebras in $\mathrm{CH}_{n}$, the module varety $X_{A}$ is a smooth affine $P G L_{n}$-variety. The coordinate ring of the quotient variety $X_{A} / P G L_{n}$ is by definition the ring of invariants $\mathbb{C}\left[X_{A}\right]^{P G L_{n}}$, which by $[8$ is equal to $\operatorname{tr}(A)=Z$. The ring-theoretical properties of $Z$ follow from the fact that $\mathbb{C}\left[X_{A}\right]$ is regular and the Hochster-Roberts result.

Let us recall a few standard facts on the quotient map

$$
\pi: X_{A} \rightarrow X_{A} / P G L_{n}=Y_{A},
$$

where $Y_{A}$ is the affine variety corresponding to the center $Z$. From invariant theory we know that points of $Y$ parametrize closed $P G L_{n}$-orbits in $X_{A}$.

By the Artin-Voigt theorem (see for example 4]) we know that closed orbits in $X_{A}$ correspond to isomorphism classes of semi-simple $n$-dimensional representations of $A$. Moreover, as $A$ is an order in a central simple algebra of dimension $n^{2}$ over $K$, there is a Zariski open subset $Y_{A z} \hookrightarrow Y_{A}$ the points of which correspond to isoclasses of simple $n$-dimensional representations of $A$. We call $Y_{A z}$ the Azumaya locus of $A$. Equivalently, if $m$ is the maximal ideal of $Z$ corresponding to a point $y \in Y$, then $y \in Y_{A z}$ if and only if $A / m A \simeq M_{n}(\mathbb{C})$. 
For an arbitrary point $y \in Y$ with corresponding maximal ideal $m$ of $Z$ we have

$$
(A / m A) / r a d \simeq M_{d_{1}}(\mathbb{C})^{\oplus e_{1}} \oplus \ldots \oplus M_{d_{r}}(\mathbb{C})^{\oplus e_{r}}
$$

with $\sum d_{i} e_{i}=n$. Here, the factors correspond to the simple components of the semi-simple representation determined by $y$; these components have dimension $d_{i}$ and occur with multiplicity $e_{i}$.

By $\tau(m)$ or $\tau(y)$ we will denote the representation type of this semi-simple $n$-dimensional representation, that is, the numerical data $\left(e_{1}, d_{1} ; \ldots ; e_{r}, d_{r}\right)$. By $Y(\tau)$ we denote the subset of $Y$ consisting of points of representation type $\tau$. In particular, $Y_{A z}=Y(1, n)$.

There is a natural order relation on the representation types given by degenerations of the corresponding representations. This order is induced by the following two operations:

$$
\begin{aligned}
& (\ldots ; e, d ; \ldots) \leq\left(\ldots ; e, d^{\prime} ; e, d^{\prime \prime} ; \ldots\right) \text { where } d=d^{\prime}+d^{\prime \prime} \\
& \left(\ldots ; e^{\prime}, d ; e^{\prime \prime}, d ; \ldots\right) \leq(\ldots ; e, d ; \ldots) \quad \text { where } e=e^{\prime}+e^{\prime \prime}
\end{aligned}
$$

This combinatorial order relation relates to the stabilizer subgroups of closed orbits.

If $\tau=\left(e_{1}, d_{1} ; \ldots ; e_{r}, d_{r}\right)$, then one verifies that the stabilizer subgroup of $G L_{n}$ in the semi-simple representation of type $\tau$ is equal to

$$
G L(\tau)=G L_{e_{1}} \times \ldots \times G L_{e_{r}}
$$

embedded via

$$
\left[\begin{array}{ccc}
G L_{e_{1}} \otimes 1_{d_{1}} & & \\
& \ddots & \\
& & G L_{e_{r}} \otimes 1_{d_{r}}
\end{array}\right] \hookrightarrow G L_{n} .
$$

Then $\tau \leq \tau^{\prime}$ if and only if $G L\left(\tau^{\prime}\right)$ is conjugate to a subgroup of $G L(\tau)$.

\section{Etale local structure}

If $X$ is a commutative smooth variety of dimension $d$ and $x$ a point of $X$, then there is only one type of étale local behaviour at $x$, namely

$$
\mathcal{O}_{x}^{s h} \simeq \mathbb{C}\left\{x_{1}, \ldots, x_{d}\right\}
$$

the strict henselization of the local ring in $x$ is the ring of algebraic functions on $d$ variables.

In this section we will prove an analogous result for smooth orders in $C H_{n}$. We will show in the next section that for fixed $n$ and $d$ (the Krull dimension of the center) there are only finitely many types of étale behaviour.

From now on we fix a smooth algebra $(A, t r)$ in $C H_{n}$, which we assume to be an order in a central simple $K$-algebra $\Delta$ of dimension $n^{2} . K$ is the field of fractions of the center $Z$ of $A$.

For $y \in Y(\tau)$ and corresponding maximal ideal $m \triangleleft Z$, by the étale local type of $A$ at $y$ we mean the structure of the algebra

$$
A_{m}^{s h}=A \otimes_{Z} Z_{m}^{s h} .
$$

The next result was proved in 10 by a different method.

Proposition 3.1. An Azumaya algebra $A$ is smooth in $C H_{n}$ if and only if its center $Z$ is smooth. 
Proof. As $A$ is an Azumaya algebra, $X_{A}$ is a principal $P G L_{n}$-bundle over $Y_{A}$. Clearly, a principal bundle is smooth if and only if the base-space is smooth.

Lemma 3.2. If $y \in Y_{A z}$, then $A_{m}^{s h} \simeq M_{n}\left(\mathbb{C}\left\{x_{1}, \ldots, x_{d}\right\}\right)$.

Proof. If $y \in Y_{A z}$, then $A \otimes_{Z} Z_{m}$ is an Azumaya algebra and hence split by an étale extension. By the foregoing proposition, $y$ is a smooth point of $Y$, and the result follows.

In general, let $m$ be the maximal ideal of $Z$ corresponding to $y \in Y(\tau)$. Fix a point $x \in X_{A}$ in the closed orbit corresponding to $y$. The semi-simple $A$-module $M_{x}$ has decomposition into simple components

$$
M_{x}=S_{1}^{\oplus e_{1}} \oplus \ldots \oplus S_{r}^{\oplus e_{r}}
$$

where $\operatorname{dim}\left(S_{i}\right)=d_{i}$ and the stabilizer $G L_{x}$ of the $G L_{n}$-action on $X_{A}$ is equal to $G L(\tau)$. By $\operatorname{Orb}_{x}$ we will denote the orbit $(P) G L_{n} . x$ of $x$ in $X_{A}$.

We have $G L_{n}$-equivariant closed embeddings

$$
\mathrm{Orb}_{x} \hookrightarrow X_{A} \hookrightarrow M_{n}(\mathbb{C})^{\oplus m}
$$

and corresponding embeddings of the tangent spaces in $x$

$$
T_{x} \operatorname{Orb}_{x} \hookrightarrow T_{x} X_{A} \hookrightarrow T_{x} M_{n}(\mathbb{C})^{\oplus m},
$$

which are embeddings as $G L(\tau)$-modules, and hence by reductivity of $G L(\tau)$ they are direct factors.

Therefore, for the normal spaces to the orbit in $X_{A}\left(\operatorname{resp} . M_{n}(\mathbb{C})^{\oplus m}\right)$ we have

$$
N_{x}^{s m}=\frac{T_{x} X_{A}}{T_{x} O r b_{x}} \triangleleft N_{x}^{b i g}=\frac{T_{x} M_{n}(\mathbb{C})^{\oplus m}}{T_{x} O r b_{x}}
$$

as $G L(\tau)$-modules. That is, we have the following picture:

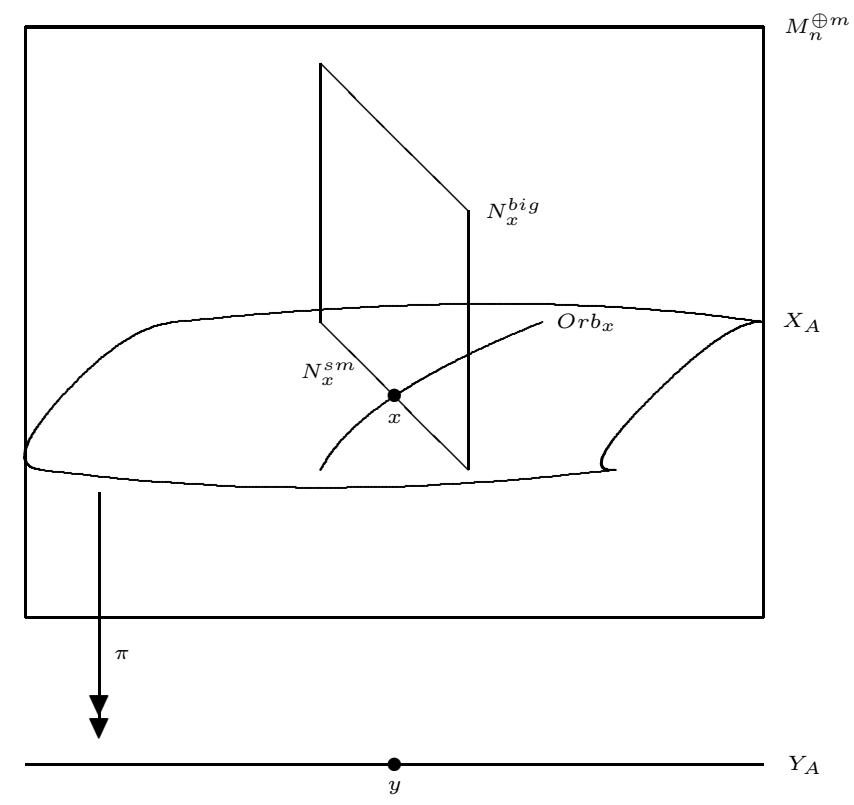

Before we compute these $G L(\tau)$-modules, let us explain the relevance to our problem. 
This is an application of the Luna slice theorem in invariant theory (see [7] or [11]) adapted to the situation of interest to us.

In general, if $H$ is a reductive subgroup of $G$ acting on an affine variety $Z$, then one defines an $H$-action on $G \times Z$ via the map

$$
h .(g, z)=\left(g h^{-1}, h . z\right) .
$$

The corresponding quotient is called the associated fiber bundle

$$
G \times{ }^{H} Z=(G \times Z) / H,
$$

and it acquires a $G$-action via multiplication on the left in the first component. One can show that the corresponding quotient satisfies

$$
\left(G \times{ }^{H} Z\right) / G \simeq Z / H
$$

Theorem 3.3 (Luna slice theorem). Let $x$ be a smooth point of $X_{A}$ of representation type $\tau$. Then, there exists a locally closed affine smooth subvariety $S_{x} \hookrightarrow X_{A}$ containing $x$, stable under the action of $G L(\tau)$ and satisfying the following properties:

- The map $G L_{n} \times S_{x} \rightarrow X_{A}$ obtained by $(g, s) \mapsto g . s$ induces a $G L_{n}$-equivariant étale map

$$
\psi: G L_{n} \times^{G L(\tau)} S_{x} \rightarrow X_{A}
$$

with affine image. Moreover, the induced quotient map

$$
\psi / G L_{n}:\left(G L_{n} \times G L(\tau) S_{x}\right) / G L_{n}=S_{x} / G L(\tau) \rightarrow X_{A} / G L_{n}=Y_{A}
$$

is also étale.

- There is a $G L(\tau)$-equivariant map

$$
\phi: S_{x} \rightarrow N_{x}^{s m}=T_{x} S_{x}
$$

such that $\phi(x)=0$ and with affine image. The induced quotient map

$$
\phi / G L(\tau): S_{x} / G L(\tau) \rightarrow N_{x}^{s m} / G L(\tau)
$$

is also étale.

- The above maps induce the following commutative diagram:

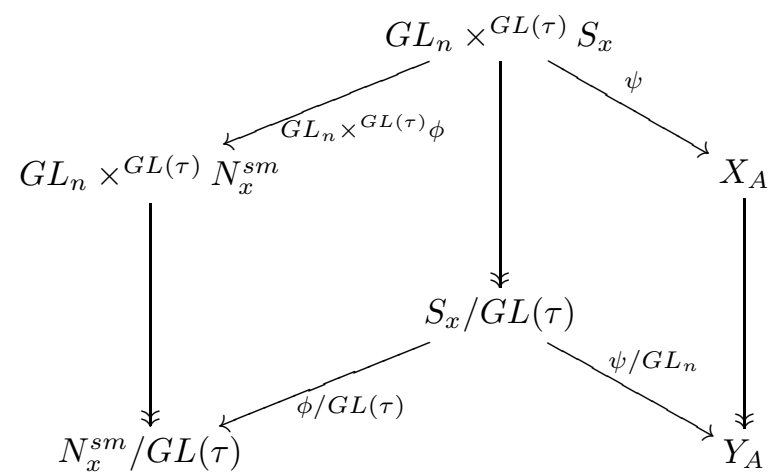

where the vertical maps are the quotient maps, all diagonal maps are étale and the upper ones are $G L_{n}$-equivariant. 
Hence, the $G L_{n}$-local structure of $X_{A}$ in a neighborhood of $x$ is the same as that of $G L_{n} \times{ }^{G L(\tau)} N_{x}^{s m}$ in a neighborhood of $\overline{\left(1_{n}, 0\right)}$. Similarly, the local structure of $Y_{A}$ in a neighborhood of $m$ is the same as that of $N_{x}^{s m} / G L(\tau)$ in a neighborhood of $\overline{0}$. Therefore, we have

Theorem 3.4. Let $A$ be a smooth order in $C H_{n}$ with center $Z$. Let $m$ be a maximal ideal of $Z$ corresponding to a point $x \in \bmod _{n} B$ of representation type $\tau=\left(e_{1}, d_{1} ; \ldots ; e_{r}, d_{r}\right)$. Let $p$ denote the maximal ideal of $\mathbb{C}\left[N_{x}^{\text {small }} / G L(\tau)\right]$ corresponding to the point $\overline{0}$. Then,

1. $Z_{m}^{s h} \simeq \mathbb{C}\left[N_{x}^{s m} / G L(\tau)\right]_{p}^{s h}$, and

2. $A_{m}^{s h} \simeq\left(M_{n}\left(\mathbb{C}\left[G L_{n} \times{ }^{G L(\tau)} N_{x}^{s m}\right]\right)^{G L_{n}}\right)_{p}^{s h}$.

Hence, we know the étale local structure of $Z$ and $A$ in $m$ if we know the $G L(\tau)$ module structure of $N_{x}^{s m}$.

Since we know the embedding $G L(\tau) \hookrightarrow G L_{n}$ and the action of $G L_{n}$ on $M_{n}(\mathbb{C})^{\oplus m}$ (by simultaneous conjugation), we know the structure of $T_{x} M_{n}(\mathbb{C})^{\oplus m}=M_{n}(\mathbb{C})^{\oplus m}$ as $G L(\tau)$-module. Further, the exact sequence

$$
0 \rightarrow \text { Lie } G L(\tau) \rightarrow \text { Lie } G L_{n} \rightarrow T_{x} \text { Orb }_{x} \rightarrow 0
$$

allows us to determine the $G L(\tau)$-module structure of $T_{x} O r b_{x}$ and consequently that of $N_{x}^{b i g}=T_{x} M_{n}(\mathbb{C})^{\oplus m} / T_{x} O r b_{x}$.

Once we know an isotypical decomposition of $N_{x}^{b i g}$, taking a direct subsum we obtain all possibilities for $N_{x}^{s m}$. Of course, later on, we will have to verify which of these theoretical possibilities actually occur for a smooth order $A$ in $C H_{n}$.

Rather than writing down decompositions of $N_{x}^{s m} \triangleleft N_{x}^{b i g}$ in simple $G L(\tau)$-modules we prefer to represent this information by a 'local chart'. We use the following dictionary:

- A loop at vertex $(i)$ corresponds to the $G L(\tau)$-module $M_{e_{i}}(\mathbb{C})$ on which $G L_{e_{i}}$ acts by conjugation and the other factors act trivially.

- An arrow from vertex $(i)$ to vertex $(j)$ corresponds to the $G L(\tau)$-module $M_{e_{i} \times e_{j}}(\mathbb{C})$ on which $G L_{e_{i}} \times G L_{e_{j}}$ acts via $g . m=g_{i} m g_{j}^{-1}$ and the other factors act trivially.

- A marked loop at vertex $(i)$ corresponds to the simple $G L(\tau)$-module $M_{e_{i}}^{0}(\mathbb{C})$, that is, trace zero matrices with action of $G L_{e_{i}}$ by conjugation and trivial action by the other components.

- The label of a loop or arrow indicates the multiplicity of the corresponding representation.

Lemma 3.5. With conventions as above and $x$ a point of representation type $\tau$, we have:

1. The $G L(\tau)=G L_{e_{1}} \times \ldots \times G L_{e_{r}}$-module structure of $N_{x}^{\text {big }}$ can be represented by the local chart on $r$ vertices such that the subchart on any two vertices $1 \leq i, j \leq r$ is of the form

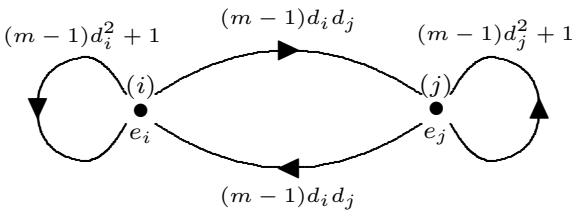


2. The $G L(\tau)$-module structure of $N_{x}^{s m}$ can be represented by a local chart on $r$ vertices such that the subgraph on any two vertices $1 \leq i, j \leq r$ is of the form

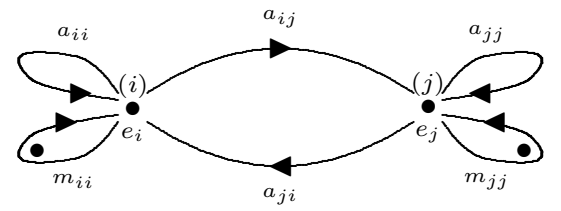

where $a_{i j} \leq(m-1) d_{i} d_{j}$ and $a_{i i}+m_{i i} \leq(m-1) d_{i}^{2}+1$ for all $1 \leq i, j \leq r$.

Proof. (2) follows from (1) by observing that $M_{e_{i} \times e_{j}}(\mathbb{C})$ is a simple $G L(\tau)$-module and that the isotypical decomposition of $M_{e_{i}}(\mathbb{C})$ is $M_{e_{i}}^{0}(\mathbb{C}) \oplus \mathbb{C}_{\text {triv }}$, where $\mathbb{C}_{\text {triv }}$ is the trivial one-dimensional $G L(\tau)$-module. The isotypical decomposition of $N_{x}^{b i g}$ has been proved in [5].

\section{Classifying local charts}

A local chart $C=(M, \mathbf{e})$ consists of two data: the underlying 'map' $M$ (that is, the marked labeled directed graph) and the 'dimension-vector' $\mathbf{e}=\left(e_{1}, \ldots, e_{r}\right)$. If we specify e we obtain a $G L(\boldsymbol{e})=\times G L_{e_{i}}$-module $R(M, \mathbf{e})$ any vector of which we call a representation of the map $M$ of dimension $\mathbf{e}$. That is, $v \in R(M, \mathbf{e})$ assigns to each

- arrow from $(i)$ to $(j)$ a matrix in $M_{e_{i} \times e_{j}}(\mathbb{C})$,

- unmarked loop in $(i)$ a matrix in $M_{e_{i}}(\mathbb{C})$,

- marked loop in $(i)$ a trace zero matrix in $M_{e_{i}}^{0}(\mathbb{C})$.

A morphism from a representation $v \in R(M, \mathbf{e})$ to a representation $w \in R(M, \mathbf{f})$ is an $r$-tuple of linear maps $\psi=\left(\psi_{1}, \ldots, \psi_{r}\right) \in \bigoplus_{i} M_{f_{i} \times e_{i}}(\mathbb{C})$ such that every diagram

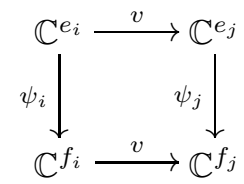

is commutative, where the horizontal maps are either arrows or (marked) loops in $M$.

Having morphisms, the notions of sub-, quotient- and simple-representation are obvious as are direct sums of representations of $M$. If we view the $G L(\mathbf{e})$-module $R(M, \mathbf{e})$ as an affine space on which $G L(\mathbf{e})$ acts, then orbits correspond precisely to isomorphism classes of representations.

Lemma 4.1. The local chart $C=(M, \mathbf{e})$ of a smooth order $A$ in $C H_{n}$ at a point $x$ must be such that $R(M, \mathbf{e})$ contains simple representations of $M$.

Proof. Consider a point $x \in X_{A}$ of representation type $\tau=\left(e_{1}, d_{1} ; \ldots ; e_{r}, d_{r}\right)$ with $N_{x}^{s m}=R(M, \mathbf{e})$. By the Luna slice theorem we have étale $G L_{n}$-equivariant maps

$$
G L_{n} \times G L(\tau) N_{x}^{s m} \stackrel{e t}{\leftarrow} G L_{n} \times \stackrel{G L(\tau)}{ } S_{x} \stackrel{e t}{\rightarrow} X_{A} .
$$

As $A$ is a prime order, we have that any Zariski neighborhood of $x$ in $X_{A}$ contains simple orbits, that is, closed orbits with stabilizer $\mathbb{C}^{*}$. Because the maps above are 
$G L_{n}$-equivariant and étale, every Zariski neighborhood of $\overline{\left(1_{n}, 0\right)}$ contains closed $G L_{n}$-orbit with stabilizer $\mathbb{C}^{*}$. By the correspondence of orbits in fiber bundles there must be closed $G L(\tau)$-orbits in $N_{x}^{s m}=R(M, \mathbf{e})$ with stabilizer $\mathbb{C}^{*}$. By a version of the Artin-Voigt theorem for representations of the map $M$, closed orbits correspond to semi-simple representations of $M$. If the stabilizer of such a representation is $\mathbb{C}^{*}$, then it must be simple.

Hence, we have to determine which dimension vectors can arise from simple representations of the map $M$. We define the Euler-form of $M$ as the bilinear map

$$
\chi_{M}: \mathbb{Z}^{r} \times \mathbb{Z}^{r} \rightarrow \mathbb{Z}
$$

determined by the matrix $\chi_{M}=\left(\chi_{i j}\right)$ with entries

$$
\chi_{i j}=-a_{i j} \quad \text { and } \quad \chi_{i i}=1-a_{i i}-m_{i i}
$$

where $a_{i j}$ is the number of arrows from $(i)$ to $(j)$ in $M$, and $a_{i i}$ resp. $m_{i i}$ are the number of (resp. marked) loops at $(i)$.

Proposition 4.2. $\mathbf{e}=\left(e_{1}, \ldots, e_{r}\right)$ is the dimension-vector of a simple representation of the map $M$ if and only if one of the following situations occurs:

1. $M=\tilde{A}_{r}$ is the extended Dynkin diagram with cyclic orientation and $\mathbf{e}=$ $(1, \ldots, 1)$.

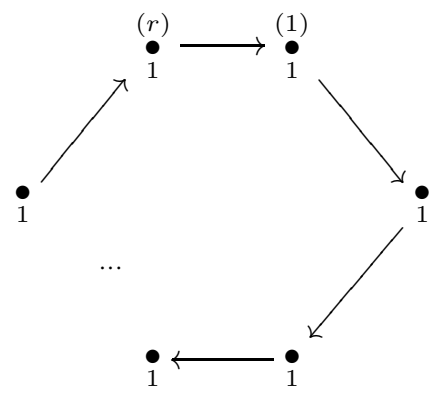

2. $M \neq \tilde{A}_{r}$. Then, $M$ has to be strongly connected (that is, any two vertices can be connected by a directed path $)$, and the if $\delta_{i}=\left(\delta_{1 i}, \ldots, \delta_{r i}\right)$ are a standard basis of $\mathbb{Z}^{r}$ we must have

$$
\chi_{M}\left(\mathbf{e}, \delta_{i}\right) \leq 0 \quad \text { and } \quad \chi\left(\delta_{i}, \mathbf{e}\right) \leq 0
$$

for all $1 \leq i \leq r$.

Proof. We will only prove necessity of the conditions in (2). Sufficiency follows from a degeneration argument and induction, as in [6].

Let $v \in R(M, \mathbf{e})$ be a simple representation (that is, containing no proper subrepresentations) and let $v(\phi)$ denote the linear map determined by the arrow, loop or marked loop $\phi$.

Assume $M$ is not strongly connected. Then we can divide $M$ into maximal strongly connected submaps, $M_{1}, \ldots, M_{z}$, say. The directions of all arrows between two such components must be all the same by maximality. Hence, there is a component $M_{i}$ having no arrows to other components. Now, define a proper subrepresentation $w$ of $v$ with dimension-vector $\mathbf{f}=\delta_{M}$.e by $w(\phi)=v(\phi)$ if $\phi$ is a map in $M_{i}$ and $w(\phi)=0$ otherwise. Hence, $M$ must be strongly connected. 
For each $(i)$ we have

$$
\chi_{M}\left(\delta_{i}, \mathbf{e}\right)=e_{i}-\sum_{(i) \stackrel{\phi}{\rightarrow}(j)} e_{j} .
$$

Hence, if $\chi_{M}\left(\delta_{i}, \boldsymbol{e}\right)>k$ then the natural morphism

$$
\bigoplus_{(i) \stackrel{\phi}{\rightarrow}(j)} v(\phi): \mathbb{C}^{e_{i}} \rightarrow \underset{(i) \stackrel{\phi}{\rightarrow}(j)}{\bigoplus} \mathbb{C}^{e_{j}}
$$

has a non-trivial kernel $K$ of dimension $k>0$ and determines a proper subrepresentation of $v$ of dimension-vector $\mathbf{f}=\left(\delta_{i} j \cdot k\right)_{j}$.

Similarly, if

$$
\chi_{M}\left(\mathbf{e}, \delta_{i}\right)=e_{i}-\sum_{(j) \stackrel{\phi}{\rightarrow}(i)} e_{j}>0,
$$

then the image of the natural morphism

$$
\bigoplus_{(j) \stackrel{\phi}{\rightarrow}(i)} v(\phi): \bigoplus_{(i) \stackrel{\phi}{\rightarrow}(j)} \mathbb{C}^{e_{j}} \rightarrow \mathbb{C}^{e_{i}}
$$

is a proper subspace of $\mathbb{C}^{e_{i}}$ of dimension $k<e_{i}$ and hence determines a proper subrepresentation of $v$ with dimension-vector $\mathbf{e}+\left(k-e_{i}\right) \delta_{i}$.

Proposition 4.3. The local chart $C=(M, \mathbf{e})$ of a smooth model $A$ in $C H_{n}$ having a center $Z$ of (Krull) dimension d must be such that

$$
1-\chi_{M}(\mathbf{e}, \mathbf{e})-\sum_{i} m_{i i}=d
$$

Proof. Consider a point $x \in X_{A}$ of representation type $\tau=\left(e_{1}, d_{1} ; \ldots ; e_{r}, d_{r}\right)$ with $N_{x}^{s m}=R(M, \mathbf{e})$. By the Luna slice theorem we have étale maps

$$
N_{x}^{s m} / G L(\tau) \leftarrow^{e t} S_{x} / G L(\tau) \rightarrow^{e t} Y_{A} .
$$

Because $\mathbb{C}\left[Y_{A}\right]=Z$ we have that $Y_{A}$ and hence $N_{x}^{s m} / G L(\tau)$ must be of dimension $d$.

By definition of the Euler-form of $M$ we have that

$$
\chi(\mathbf{e}, \mathbf{e})=-\sum_{i \neq j} e_{i} e_{j} a_{i j}+\sum_{i} e_{i}^{2}\left(1-a_{i i}-m_{i i}\right) .
$$

On the other hand, we have the following dimensions:

$$
\begin{gathered}
\operatorname{dim} R(M, \mathbf{e})=\sum_{i \neq j} e_{i} e_{j} a_{i j}+\sum_{i} e_{i}^{2}\left(a_{i i}+m_{i i}\right)-\sum_{i} m_{i i} \\
\operatorname{dim} G L(\mathbf{e})=\sum_{i} e_{i}^{2}
\end{gathered}
$$

As $\mathbf{e}$ is the dimension vector of a simple representation, we know that the orbits in general position in $R(M, \mathbf{e})$ are closed and have stabilizer $\mathbb{C}^{*}$. Therefore, the dimension of the quotient variety $R(M, \mathbf{e}) / G L(\mathbf{e})=N_{x}^{s m} / G L(\tau)$ is equal to

$$
\operatorname{dim} R(M, \mathbf{e})-\operatorname{dim} G L(\mathbf{e})+1
$$

and plugging in the above information we see that this is equal to $1-\chi(\mathbf{e}, \mathbf{e})-$ $\sum_{i} m_{i i}$. 
If we want to study the local structure of smooth orders $A$ in $C H_{n}$ having a center of dimension $d$, we have to compile a list of admissible charts. We will give the first steps in such a classification.

The basic idea that we use is to shrink a chart to its simplest form and classify these simplest forms for given $d$. By shrinking we mean the following process. Assume $\mathbf{e}$ is the dimension vector of a simple representation of $M$, and let $(i)$ and $(j)$ be two connected vertices with $e_{i}=e_{j}=e$. That is, we have locally the following situation:

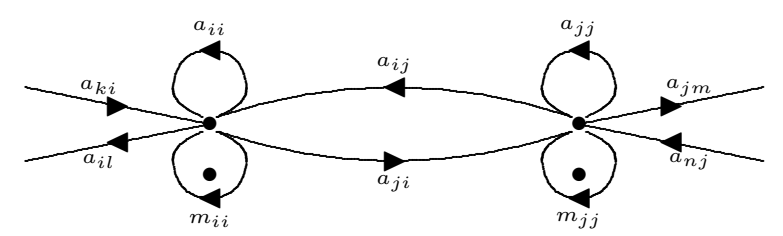

We will use one of the arrows connecting $(i)$ with $(j)$ to identify the two vertices. That is, we form the shrinked chart $C^{s}=\left(M^{s}, \mathbf{e}^{\mathbf{s}}\right)$, where $M^{s}$ is a map on $r-1$ vertices $\{(1), \ldots,(\hat{i}), \ldots,(r)\}$ and $\mathbf{e}^{\mathbf{s}}$ is the dimension vector with $(i)$ removed. That is, locally round $z$ the shrinked chart has the form

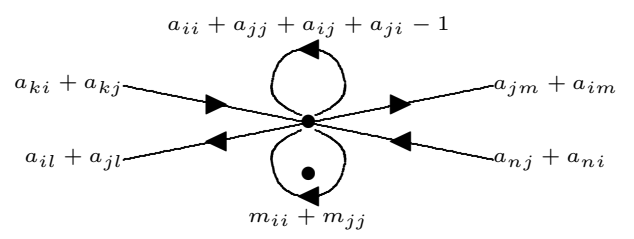

That is, in $M^{s}$ we have for all $k, l \neq j$ that $a_{k l}^{s}=a_{k l}$. Moreover, the number of arrows and (marked) loops connected to $j$ are determined as follows:

- $a_{j k}^{s}=a_{i k}+a_{j k}$

- $a_{k j}^{s}=a_{k i}+a_{k j}$

- $a_{j j}^{s}=a_{i i}+a_{j j}+a_{i j}+a_{j i}-1$,

- $m_{j j}^{s}=m_{i i}+m_{j j}$.

Lemma 4.4. $\mathrm{e}$ is the dimension vector of a simple representation of $M$ if and only if $\mathbf{e}^{\mathbf{s}}$ is the dimension vector of a simple representation of $M^{s}$. Moreover,

$$
\operatorname{dim} R(M, \mathbf{e}) / G L(\mathbf{e})=\operatorname{dim} R\left(M^{s}, \mathbf{e}^{\mathbf{s}}\right) / G L\left(\mathbf{e}^{\mathbf{s}}\right)
$$

Proof. Fix an arrow $\phi$ connecting $(i)$ and $(j)$. As $e_{i}=e_{j}=e$, there is a Zariski open subset $U \hookrightarrow R(M, \mathbf{e})$ of points $v$ such that $v(\phi)$ is invertible. By basechange in either $(i)$ or $(j)$, we can find a point $w$ in its orbit such that $w(\phi)=I_{e}$.

If we think of $w(\phi)$ as identifying $\mathbb{C}^{e_{i}}$ with $\mathbb{C}^{e_{j}}$ we can view the remaining maps of $w$ as a representation in $R\left(M^{s}, \mathbf{e}^{\mathbf{s}}\right)$ and denote it by $w^{s}$. The map $U \rightarrow R\left(M^{s}, \mathbf{e}^{\mathbf{s}}\right)$ is well-defined and maps $G L(\mathbf{e})$-orbits to $G L\left(\mathbf{e}^{\mathbf{s}}\right)$-orbits.

Conversely, given a representation $w^{\prime} \in R\left(M^{s}, \mathbf{e}^{\mathbf{s}}\right)$, we can uniquely determine a representation $w \in U$ mapping to $w^{\prime}$.

Both claims follow immediately from this observation.

It is clear that any chart can uniquely be reduced to its simplest form, which has the property that no connecting vertices can have the same dimension. Also note 
that the shrinking process has a not necessarily unique converse operation, which we will call splitting of a vertex.

Proposition 4.5. Let $\mathbf{e}$ be the dimension vector of a simple representation of $M$, and let $d=\operatorname{dim} R(M, \mathbf{e}) / G L(\mathbf{e})$. If $e=\max e_{i}$, then $d \geq e+1$.

Proof. By the above lemma we may assume that $C=(M, \mathbf{e})$ is in its simplest form, that is, no two connecting vertices have same dimension. Let $\chi_{M}=I_{r}-\left(g_{i j}\right)_{i, j}$, where $g_{i j}$ is the number of arrows (or loops, marked or unmarked) from $(i)$ to $(j)$. We can then rewrite

$$
-\chi_{M}(\mathbf{e}, \mathbf{e})=\sum_{i} e_{i}\left(\sum_{j} g_{i j} e_{j}-e_{i}\right)=\sum_{i} e_{i}\left(\sum_{j} g_{j i} e_{j}-e_{i}\right)
$$

and observe that the terms between brackets are positive, thanks to the requirement that $\mathbf{e}$ is the dimension vector of a simple representation. For any vertex $(k)$ we will call

$$
e_{k}\left(\sum_{l \neq k} a_{l k} e_{l}+\left(a_{k k}+m_{k k}-1\right) e_{k}\right)-m_{k k}
$$

and

$$
e_{k}\left(\sum_{l \neq k} a_{k l} e_{l}+\left(a_{k k}+m_{k k}-1\right) e_{k}\right)-m_{k k}
$$

the incoming (resp. outgoing) contribution of $(k)$ to $\operatorname{dim} R(M, \mathbf{e}) / G L(\mathbf{e})$.

Observe that the in (out) contributions of vertices having no marked loops is always $\geq 0$ by the restrictions on e. Further, if there are marked loops at a vertex $(i)$, then the in (out) contribution of that vertex is $\geq 1$. For, it is

$$
e_{i}\left(\left(a_{i i}+m_{i i}-1\right) e_{i}+\sum_{j} a_{i j} e_{j}\right)-m_{i i} \geq a_{i i}+m_{i i}+1-m_{i i} \geq 1
$$

(note that $e_{i} \neq e_{j}$ ).

Consider a vertex $(z)$ where the maximum dimesion vector $e$ is obtained. We have locally the following situation:

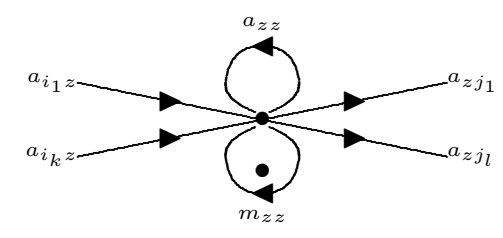

The out-contribution of $(z)$ is equal to

$$
e\left(\left(a_{z z}+m_{z z}-1\right) e+\sum_{u} a_{z j_{u}} e_{j_{u}}\right)-m_{z z} .
$$

If $m_{z z} \geq 2$ this expression is $\geq e^{2}\left(m_{z z}-1\right)+2-m_{z z} \geq e$ because $e \geq 2$. Hence, the dimension is at least $a+1$.

If $m_{z z}=1$ then the in-contribution of $(z)$ is $\geq e-1$ and the in-contributions of $j_{u}$ are $\geq e-1$, so the total dimension is $\geq 2 e-1 \geq e+1$ if $e \geq 2$.

Finally, if $m_{z z}=0$ then the out-contribution of $(z)$ is

$$
\geq e\left(\sum_{u} a_{z j_{u}} e_{j_{u}}-e\right)
$$


which is $\geq e$ whenever $\sum_{u} a_{z j_{u}} e_{j_{u}} \neq a$. If this happens to be an equality, then the in-contribution at $j_{u}$ is

$$
\geq e_{j_{u}}\left(\left(m_{j_{u} j_{u}}-1\right) e_{j_{u}}+e\right)-m_{j_{u} j_{u}} \geq e-1
$$

but there are at least two $j_{u}$ as none of the $e_{j_{u}}=e$, so again the dimension $\geq 2 e-1 \geq e+1$.

Definition 4.6. Two charts $C=(M, \mathbf{e})$ and $C^{\prime}=(M, \mathbf{e})$ are said to be equivalent if their corresponding $G L(\mathbf{e})$-modules are isomorphic.

Example 4.7. The charts below are equivalent:
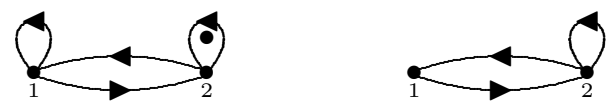

Theorem 4.8. The local charts of a smooth order $A$ in $C H_{n}$ with center $Z$ of Krull dimension $d$ can be shrunk to one of the following equivalence classes of charts:
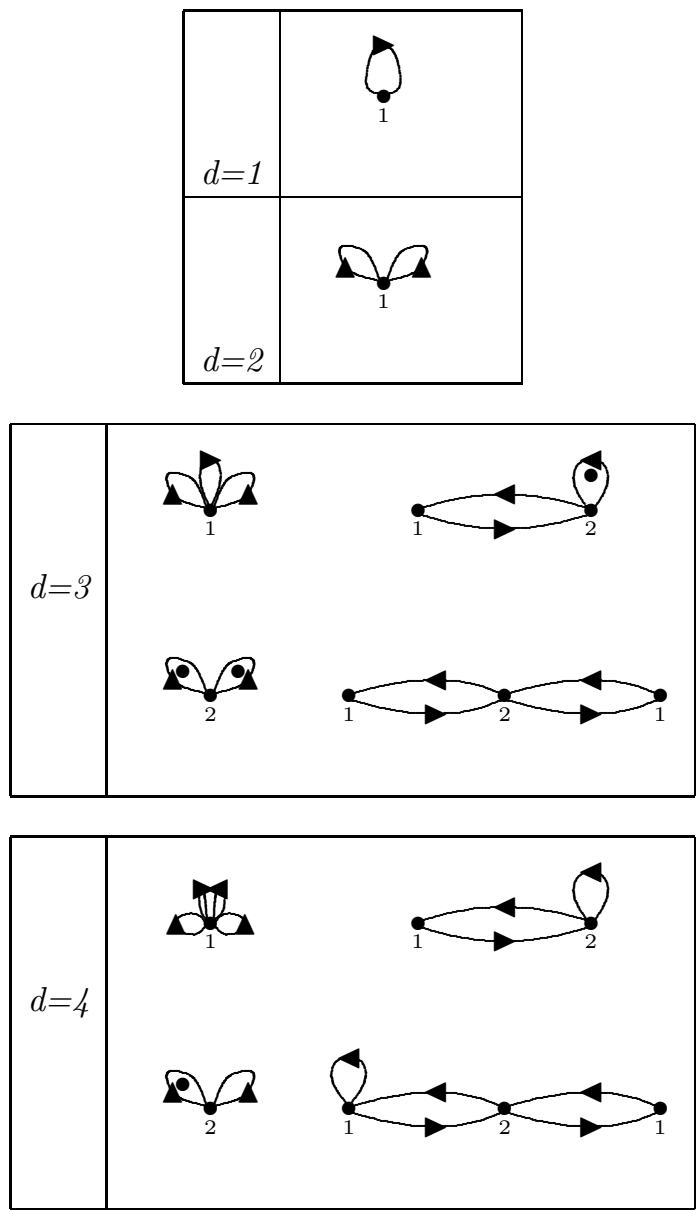

It is clear that one can easily compile a list of equivalence classes of simplified charts for any given dimension $d$. A direct consequence is 
Proposition 4.9. For fixed $n$ and $d$ there are only finitely many types of étale local behaviour.

Proof. By the foregoing reduction there are for fixed $d$ only finitely many equivalence classes of local charts shrunk to their simplest form. As $\sum e_{i} \leq n$, we can only apply splitting of vertices finitely many times.

\section{READing the LOCAL CHART}

Knowing which local charts can occur, we will now investigate what information can be derived from the local chart.

We will fix the following situation: $A$ is a smooth order in $C H_{n}$ having a center $Z$ of Krull dimension $d$, and $m$ will be a maximal ideal of $Z$ corresponding to the closed orbit $G L_{n} . x \hookrightarrow X_{A}$, where $x$ has representation type $\tau=\left(e_{1}, d_{1} ; \ldots ; e_{r}, d_{r}\right)$.

We have $N_{x}^{s m}=R(M, \mathbf{e})$ as $G L(\tau)=\times G L_{e_{i}}$ - module. The local structure of $Z$ near $m$ is determined by that of $\mathbb{C}\left[N_{x}^{s m} / G L_{\tau}\right]$ near the zero representation, so we have an interpretation of this ring

Proposition 5.1. $\mathbb{C}\left[N_{x}^{s m} / G L(\tau)\right]$ is generated by traces along oriented cycles in the chart $C=(M, \mathbf{e})$.

That is, for every arrow $\phi$ (resp. loop or marked loop) from $(i)$ to $(j)$ we take a generic rectangular matrix

$$
M_{\phi}=\left[\begin{array}{cccc}
x_{11}(\phi) & \ldots & \ldots & x_{1, e_{j}}(\phi) \\
\vdots & & & \vdots \\
x_{e_{i} 1}(\phi) & \ldots & \ldots & x_{e_{i} e_{j}}(\phi)
\end{array}\right]
$$

(resp. a generic square matrix or generic trace zero matrix).

If $c y c=\phi_{k} \circ \ldots \circ \phi_{2} \circ \phi 1$ is an oriented cycle in the map $M$, then we compute the matrix

$$
M_{c y c}=M_{\phi_{k}} \ldots . M_{\phi_{2}} \cdot M_{\phi_{1}}
$$

over $\mathbb{C}\left[x_{k l}(\phi)\right]=\mathbb{C}[R(M, \mathbf{e})]$. If the starting vertex of $\phi_{1}$ is $(i)$, then this is a square $e_{i} \times e_{i}$ matrix and we can consider its trace

$$
\operatorname{Tr}\left(M_{c y c}\right) \in \mathbb{C}[R(M, \mathbf{e})],
$$

and one verifies easily that this polynomial is invariant under the action of $G L_{\tau}$. Slightly harder to prove is that these functions actually generate

$$
\mathbb{C}[R(M, \mathbf{e})]^{G L_{\tau}}=\mathbb{C}\left[N_{x}^{s m} / G L_{\tau}\right] .
$$

The essential ingredient in this proof is the fact that the polynomial invariants of tuples of matrices under simultaneous conjugation are generated by traces of products of generic matrices.

In fact, one can even bound the length of the oriented cycles to be considered by $\left(\sum_{i} e_{i}\right)^{2}$. See [6] for more details.

Next, let us consider the étale local structure of $A$ near $m$. By the results proved before, we have to control for this the ring of $G L_{n}$-equivariant maps

$$
G L_{n} \times{ }^{G L_{\tau}} R(M, \mathbf{e}) \rightarrow M_{n}(\mathbb{C}),
$$

on which the multiplication is given by that in target space $M_{n}(\mathbb{C})$. 
Proposition 5.2. The ring of $G L_{n}$-equivariant maps is Morita equivalent to the ring of $G L_{\tau}$-equivariant maps

$$
R(M, \mathbf{e}) \rightarrow M_{\sum e_{i}}(\mathbb{C}),
$$

where for any two vertices $(i)$ and $(j)$ the $G L_{\tau}$-equivariant maps

$$
R(M, \mathbf{e}) \rightarrow \operatorname{Hom}\left(\mathbb{C}^{\oplus e_{i}}, \mathbb{C}^{\oplus e_{j}}\right)
$$

are generated as a module over $\mathbb{C}\left[N_{x}^{s m} / G L(\tau)\right]$ by the paths in the map $M$ starting from $(i)$ and ending at $(j)$.

Again, if path $=\phi_{k} \circ \ldots \circ \phi_{1}$ is such a path, then the corresponding module element is $M_{\text {path }}$. This result follows from a minor adaptation to existing results on invariants and concomitants of representations of quivers proved by C. Procesi and myself, see [6].

Apart from allowing us to compute the local structure of $Z$ and $B$ near $m$, the local chart $N_{x}^{s m}=R(M, \mathbf{e})$ also allows us to describe the local charts at nearby points and the dimensions of subvarieties of points having a specific local chart.

The points $\zeta$ in the quotient variety $N_{x}^{s m} / G L(\tau)=R(M, \mathbf{e}) / G L(\mathbf{e})$ are in oneto-one correspondence with the isomorphism classes of semi-simple representations of the map $M$ of dimension vector $\mathbf{e}$.

If $V_{\zeta}$ is a representative in the closed orbit corresponding to $\zeta$, then we can decompose $V_{\zeta}$ into its simple representations

$$
V_{\zeta}=W_{1}^{\oplus m_{1}} \oplus \ldots \oplus W_{k}^{\oplus m_{k}},
$$

where $W_{i}$ is a simple representation of the map $M$ of dimension vector $\mathbf{b}_{\mathbf{i}}$ and occurring in $V_{\zeta}$ with multiplicity $m_{i}$.

Extending previous terminology, we will say that $V_{\zeta}($ or $\zeta$ ) is of representation type $\sigma=\left(m_{1}, \mathbf{b}_{\mathbf{1}} ; \ldots ; m_{k}, \mathbf{b}_{\mathbf{k}}\right)$.

As we have a combinatorial description of all simple dimension vectors for $M$, we can determine which representation types can occur for a given $\mathbf{e}$.

By $V_{\sigma}$ we will denote the set of all points $\zeta \in N_{x}^{s m} / G L_{\tau}$ of representation type $\sigma$.

Proposition 5.3. $\left\{V_{\sigma}: \sigma\right.$ a representation type for $\left.\mathbf{e}\right\}$ is a finite stratification of the quotient variety $N_{x}^{s m} / G L(\tau)$ into locally closed irreducible smooth subvarieties. Moreover, the dimension of the stratum $V_{\sigma}$ determined by $\sigma=\left(m_{1}, \mathbf{b}_{\mathbf{1}} ; \ldots ; m_{k}, \mathbf{b}_{\mathbf{k}}\right)$ is equal to

$$
\sum_{j=1}^{k}\left(1-\chi_{M}\left(\mathbf{b}_{\mathbf{j}}, \mathbf{b}_{\mathbf{j}}\right)\right)-\sum_{i=1}^{r} m_{i i} .
$$

Proof. According to the Luna slice results, we have to verify that the representation type determines the stabilizer subgroup of a point in the closed orbit up to conjugation in $G L(\mathbf{e})$.

Let $\mathbf{b}_{\mathbf{i}}=\left(b_{i 1}, \ldots, b_{i r}\right)$ and $b_{i}=\sum_{j} b_{i j}$. We choose a basis in $\bigoplus_{i} \mathbb{C}^{\oplus e_{i}}$ in the following way: the first $m_{1} b_{1}$ vectors give a basis for the simple components of type $W_{1}$, the next $e_{2} b_{2}$ vectors give a basis for the simple components of type $W_{2}$, and so on. 
If $m=\sum a_{i}$, the subring of $M_{m}(\mathbb{C})$ generated by the representation $V_{\zeta}$ expressed in this basis is

$$
\left[\begin{array}{lll}
M_{b_{1}}(\mathbb{C}) \otimes I_{e_{1}} & & \\
& \ddots & \\
& & M_{b_{k}}(\mathbb{C}) \otimes I_{e_{k}}
\end{array}\right] .
$$

Therefore, the stabilizer $G L_{V}$ in $G L(\mathbf{e})$ of $V_{\zeta}$ is the group of units of the centralizer of this ring and is therefore equal to $G L_{m_{1}} \times \ldots \times G L_{m_{k}}$, which is embedded in $G L(\mathbf{e})$ with respect to the chosen basis as

$$
\left[\begin{array}{lll}
G L_{m_{1}}\left(\mathbb{C} \otimes I_{b_{1}}\right) & & \\
& \ddots & \\
& & G L_{m_{k}}\left(\mathbb{C} \otimes I_{b_{k}}\right)
\end{array}\right] .
$$

It is easy to see that the conjugacy class of $G L_{V}$ depends only on the representation type $\tau$.

Finally, we have seen before that the dimension of the variety of isoclasses of simple representations of $M$ of dimension vector $\mathbf{b}_{\mathbf{j}}$ is equal to $1-\chi\left(\mathbf{b}_{\mathbf{j}}, \mathbf{b}_{\mathbf{j}}\right)-\sum_{i} m_{i i}$ from which the claim about the dimension of the stratum follows.

Given two representations types $\sigma$ and $\sigma^{\prime}$, the stratum $V_{\sigma^{\prime}}$ lies in the closure of the stratum $V_{\sigma}$ if and only if the stabilizer subgroup $G L_{\sigma}$ is conjugate to a subgroup of $G L_{\sigma}$ in $G L(\mathbf{e})$. Again, mimicking similar results for representations of quivers, we can give a combinatorial solution to this problem.

Two representation types

$$
\sigma=\left(m_{1}, \mathbf{b}_{\mathbf{1}} ; \ldots ; m_{k}, \mathbf{b}_{\mathbf{k}}\right) \text { and } \sigma^{\prime}=\left(m_{1}^{\prime}, \mathbf{b}_{\mathbf{1}}{ }^{\prime} ; \ldots ; m_{k^{\prime}}^{\prime}, \mathbf{b}_{\mathbf{k}^{\prime}}^{\prime}\right)
$$

are said to be direct successors $\sigma<\sigma^{\prime}$ if and only if either

- (splitting one simple type) $k^{\prime}=k+1$ and for all but one $1 \leq i \leq k$ we have $\left(m_{i}, \mathbf{b}_{\mathbf{i}}\right)=\left(m_{j}^{\prime}, \mathbf{b}_{\mathbf{j}}^{\prime}\right)$ for a uniquely determined $j$, and for the remaining $i$ we have corresponding to it $\left(m_{i}, \mathbf{b}_{\mathbf{u}}^{\prime} ; m_{i}, \mathbf{b}_{\mathbf{v}}^{\prime}\right)$ with $\mathbf{b}_{\mathbf{i}}=\mathbf{b}_{\mathbf{u}}^{\prime}+\mathbf{b}_{\mathbf{v}}^{\prime}$; or

- (combining two simple types) $k^{\prime}=k-1$ and for all but one $1 \leq i \leq k^{\prime}$ we have $\left(m_{i}^{\prime}, \mathbf{b}_{\mathbf{i}}^{\prime}\right)=\left(m_{j}, \mathbf{b}_{\mathbf{j}}\right)$ for a uniquely determined $j$ and for the remaining $i$ we have corresponding to it $\left(m_{u}, \mathbf{b}_{\mathbf{i}}^{\prime} ; m_{v}, \mathbf{b}_{\mathbf{i}}^{\prime}\right)$ with $m_{u}+m_{v}=m_{i}^{\prime}$

The direct successor relation $<$ induces an ordering which we denote by $\ll$.

Proposition 5.4. The stratum $V_{\sigma^{\prime}}$ lies in the closure of the stratum $V_{\sigma}$ if and only if $\sigma \ll \sigma^{\prime}$.

Finally, we want to understand the étale local structure of the quotient variety $N_{x}^{s m} / G L(\tau)$ in a neighborhood of a point $\zeta \in V_{\sigma}$. This again is an application of the Luna slice results.

So, let $V$ be a semi-simple representation of $M$ corresponding to $\zeta \in V_{\sigma}$ with stabilizer subgroup $G L_{\sigma}=G L_{m_{1}} \times \ldots \times G L_{m_{k}}$. We have to investigate the $G L_{\sigma^{-}}$ module structure of the normal space to the orbit of $V$.

The tangent space to the $G L(\mathbf{e})$ orbit of $V$ is equal to the image of the natural linear map

$$
\text { Lie } G L(\mathbf{e}) \rightarrow R(M, \mathbf{e})
$$


sending an element $y \in$ Lie $G L(\mathbf{e})$ to the representation determined by the commutator $[y, V]=y \cdot V-V \cdot y \in M_{m}(\mathbb{C})$, where as above $m=\sum e_{i}$ and all embeddings are with respect to the choice of basis we introduced in the proof of proposition.

The kernel of the above map is the centralizer of the subalgebra of $M_{m}(\mathbb{C})$ generated by the representation $V$, that is, the algebra

$$
C_{V}=\left[\begin{array}{lll}
M_{m_{1}}\left(\mathbb{C} \otimes I_{b_{1}}\right) & & \\
& \ddots & \\
& & M_{m_{r}}\left(\mathbb{C} \otimes I_{b_{r}}\right)
\end{array}\right] .
$$

We thus have an exact sequence of $G L_{\sigma}$-modules

$$
0 \rightarrow C_{V} \rightarrow \text { Lie } G L(\mathbf{e}) \rightarrow T_{V} \operatorname{Orb}_{V} \rightarrow 0,
$$

where the action of $G L_{\sigma}$ is given by conjugation in $M_{m}(\mathbb{C})$ via the embedding given before.

A typical element $\gamma \in G L_{\sigma}=G L_{m_{1}} \times \ldots \times G L_{m_{k}}$ will be written as $\left(\gamma_{1}, \ldots, \gamma_{k}\right)$, and we will express the actions in terms of the $\gamma_{i}$.

$C_{V}$ as $G L_{\sigma}$-module consists of

- one $m_{1}^{2}$-dimensional representation with $\gamma_{1}^{-1} \cdot \gamma_{1}$-action,

- one $m_{k}^{2}$-dimensional representation with $\gamma_{k}^{-1} \cdot \gamma_{k}$-action.

Moreover, using our notation $\mathbf{b}_{\mathbf{i}}=\left(b_{i 1}, \ldots, b_{i r}\right)$ we have that Lie $G L(\mathbf{e})$ as $G L_{\sigma^{-}}$ module consists of

- $\sum_{j=1}^{k} b_{1 j}^{2}$ times the $m_{1}^{2}$-dimensional representation with $\gamma_{1}^{-1} \cdot \gamma_{1}$-action,

- $\sum_{j=1}^{k} b_{k j}^{2}$ times the $m_{k}^{2}$-dimensional representation with $\gamma_{k}^{-1} \cdot \gamma_{k}$-action,

- $\sum_{j=1}^{k} b_{1 j} b_{2 j}$ times the $m_{1} \times m_{2}$-dimensional representation with $\gamma_{1}^{-1} \cdot \gamma_{2}$-action,

- $\sum_{j=1}^{k} b_{k j} b_{k-1 j}$ times the $m_{k} \times m_{k-1}$-dimensional representation with $\gamma_{k}^{-1}$ .$\gamma_{k-1}$-action.

Hence, we know the $G L_{\sigma}$-module structure of $T_{V} O r b_{V}$. Next, we have to determine the $G L_{\sigma}$-module structure of $R(M, \mathbf{e})$. There are

- $b_{1 i} b_{1 j}$ times the $m_{1} \times m_{1}$-dimensional representation with $\gamma_{1}^{-1} \cdot \gamma_{1}$-action,

- $b_{1 i} b_{2 j}$ times the $m_{1} \times m_{2}$-dimensional representation with $\gamma_{1}^{-1} \cdot \gamma_{2}$-action,

- $b_{k i} b_{k j}$ times the $m_{k} \times m_{k}$-dimensional representation with $\gamma_{k}^{-1} \cdot \gamma_{k}$-action.

For each unmarked loop in $(i)$ we have the same decomposition as above, replacing all occurrences of $j$ with $i$. For a marked loop in $(i)$ we have to replace the terms of dimension $m_{i} \times m_{i}$ by

- $b_{l i}^{2}$ times the $\left(m_{i}^{2}-1\right)$-dimensional representation of trace zero matrices with $\gamma_{l}^{-1} \cdot \gamma_{l}$-action. 
We now have all the information on the $G L_{\sigma}$-module structure on the normal space to the orbit using the (split) exact sequence of $G L_{\sigma}$-modules

$$
0 \rightarrow T_{V} \operatorname{Orb}_{V} \rightarrow R(M, \mathbf{e}) \rightarrow N_{V} \rightarrow 0,
$$

and we obtain

Proposition 5.5. The étale local structure of $N_{x}^{s m} / G L(\tau)$ near $\zeta \in V_{\sigma}$ is determined by a local chart $C_{\sigma}=\left(M_{\sigma}, \mathbf{e}_{\sigma}\right)$, where $M_{\sigma}$ has $k$ vertices $\{(1), \ldots,(k)\}$, and there are

- $-\chi_{M}\left(\mathbf{b}_{\mathbf{i}}, \mathbf{b}_{\mathbf{j}}\right)$ directed arrows from $(i)$ to $(j)$ when $i \neq j$,

- $1-\chi_{1}\left(\mathbf{b}_{\mathbf{i}}, \mathbf{b}_{\mathbf{i}}\right)$ unmarked loops in $(i)$,

- $-\chi_{2}\left(\mathbf{b}_{\mathbf{i}}, \mathbf{b}_{\mathbf{i}}\right)$ marked loops in $(i)$.

where $\chi_{1}=\left(\delta_{i j}-a_{i j}\right)_{i, j}$ and $\chi_{2}=\left(-\delta_{i j} m_{i i}\right)_{i, j}$ and $\chi_{M}=\chi_{1}+\chi_{2}$. Moreover, the dimension vector $\mathbf{e}_{\sigma}=\left(m_{1}, \ldots, m_{k}\right)$.

\section{LOW DIMENSIONAL CASES}

In this section we give an alternative proof of the local classification of smooth orders in $\mathrm{CH}_{n}$ in dimensions one and two, and draw some conclusions.

In [10] W. Schelter proved that in dimension one smooth orders are hereditary. We will show that this also follows immediately from our local description. The result below also follows from reversing the shrinking process of local charts and the classification given before.

Proposition 6.1. The local charts $C=(M, \mathbf{e})$ for a smooth order $A$ in $C H_{n}$ with center of dimension one are such that $\mathbf{e}=(1, \ldots, 1)$ and the map $M$ has the form $\tilde{A}_{r}$ :

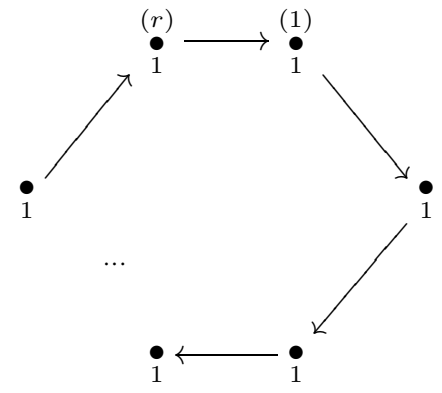

Proof. Let $(M, \mathbf{e})$ be a local chart of $A$ on $r$ vertices. As $M$ is strongly connected, there exist oriented cycles in $M$. Fix, one such cycle (say of length $s$ ) and number the vertices of $M$ so that the first $s$ vertices are those making up the cycle. Now, assume the dimension vector is $\mathbf{e}=\left(e_{1}, \ldots, e_{r}\right)$. Then, there is a semi-simple representation in $R(M, \mathbf{e})$ with composition

$$
(\underbrace{1, \ldots, 1}_{s}, \underbrace{0, \ldots, 0}_{r-s}) \oplus \delta_{s}^{\oplus e_{s}-1} \oplus \delta_{1}^{\oplus e_{1}-1} \oplus \ldots \oplus \delta_{s+1}^{\oplus e_{s+1}} \oplus \ldots \oplus \delta_{r}^{\oplus e_{r}},
$$

where $\delta_{i}$ are the standard basis of $\mathbb{Z}^{r}$, or equivalently the dimension vector of the simple representation concentrated at vertex $(i)$.

There is a one-parameter family of isoclasses of such semi-simple representations. As $\operatorname{dim} R(M, \mathbf{e}) / G L(\mathbf{e})=1$, and $\mathbf{e}$ is the dimension vector of a simpe representation, 
this can only happen if the above semi-simple representation is actually simple. Hence, $M$ is one cycle (that is, $r=s$ ) and all $e_{i}=1$.

In particular, the only representation types that can occur are of the form $\tau=$ $\left(1, d_{1}, \ldots, 1, d_{r}\right)$ and $G L(\tau)=\mathbb{C}^{*} \times \ldots \times \mathbb{C}^{*}$ embedded in $G L_{n}$ via

$$
\left(\lambda_{1}, \ldots, \lambda_{r}\right) \mapsto \operatorname{diag}(\underbrace{\lambda_{1}, \ldots, \lambda_{1}}_{d_{1}}, \ldots, \underbrace{\lambda_{r}, \ldots, \lambda_{r}}_{d_{r}}) .
$$

One verifies that

$$
\mathbb{C}[R(M, \mathbf{e}) / G L(\mathbf{e})] \simeq \mathbb{C}[x]
$$

where $x$ is the trace along the cycle. Furthermore, the ring of equivariant maps

$$
M_{n}\left(\mathbb{C}\left[G L_{n} \times{ }^{G L(\mathbf{e})} R(M, \mathbf{e})\right]\right)^{G L_{n}}
$$

has the following block decomposition:

$\left[\begin{array}{c|c|c|c}M_{d_{1}}(\mathbb{C}[x]) & M_{d_{1} \times d_{2}}(\mathbb{C}[x]) & \cdots & M_{d_{1} \times d_{r}}(\mathbb{C}[x]) \\ \hline M_{d_{2} \times d_{1}}(x \mathbb{C}[x]) & M_{d_{2}}(\mathbb{C}[x]) & \cdots & M_{d_{2} \times d_{r}}(\mathbb{C}[x]) \\ \hline \vdots & \vdots & \ddots & \vdots \\ \hline M_{d_{r} \times d_{1}}(x \mathbb{C}[x]) & M_{d_{r} \times d_{2}}(x \mathbb{C}[x]) & \cdots & M_{d_{r}}(\mathbb{C}[x])\end{array}\right]$

From this local information we deduce

Theorem 6.2. The following assertions are equivalent:

- $A$ is a smooth order in $\mathrm{CH}_{n}$ with one-dimensional center.

- $A$ is a hereditary order over a Dedekind domain.

Proof. From the local description of a smooth order given before we deduce that the center must be smooth, hence a Dedekind domain, and that the smooth order $A$ must be locally hereditary. Conversely, from the local description of hereditary orders as given in [9, Thm. 39.14] we deduce that hereditary orders are locally smooth.

In dimension two we can also give an alternative proof of our local characterization of smooth orders.

Proposition 6.3. For a smooth order $A$ in $\mathrm{CH}_{n}$ with two-dimensional center the local charts $C=(M, \mathbf{e})$ are such that $\mathbf{e}=(1, \ldots, 1)$, and the map $M$ has the 
following form:

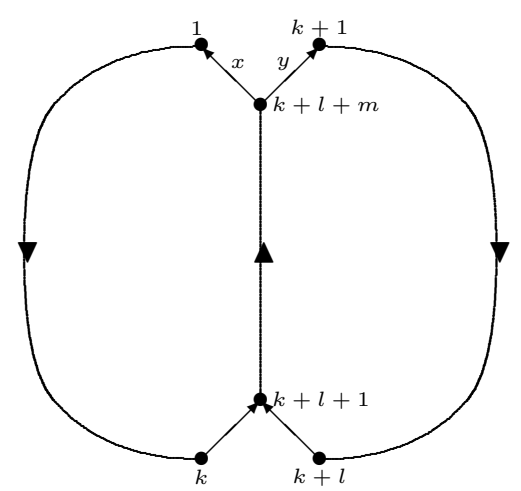

$A_{k l m}$

where the indicated numbering of vertices and labeling of arrows will be used later. In this picture we make the obvious changes whenever $k$ or $l$ is zero.

Proof. The strongly connected map $M$ must contain more than one oriented cycle, and hence contains a submap of the indicated type (possibly degenerate). It is easy to verify that, for $A_{k l m}, \mathbf{f}=(1, \ldots, 1)$ is the dimension vector of a simple representation.

If $M$ contains additional vertices $\{s=k+l+m+1, \ldots, r\}$ and/or the dimension vector $\mathbf{e}=\left(e_{1}, \ldots, e_{r}\right) \neq \mathbf{f}$, there exist semi-simple representations in $R(M, \mathbf{e})$ with dimension-vector decomposition

$$
(\underbrace{1, \ldots, 1}_{k+l+m}, 0, \ldots, 0) \oplus \delta_{1}^{e_{1}-1} \oplus \ldots \oplus \delta_{s-1}^{e_{s-1}-1} \oplus \delta_{s}^{e_{s}} \oplus \ldots \oplus \delta_{r}^{\oplus e_{r}}
$$

As $\operatorname{dim} R\left(A_{k l m}, \mathbf{f}\right) / G L(\mathbf{f})$ is equal to 2 , there is a two-dimensional family of such semi-simple representations. Hence, they cannot be properly semi-simple, as their locus must be of dimension $<d=2$. Therefore, $M=A_{k l m}$ and $\mathbf{e}=\mathbf{f}$.

Let $m$ be a maximal ideal of the center $Z$ of $A$ corresponding to a semi-simple $n$ dimensional $A$-module $M_{x}$. By the above characterization we know that $M_{x}$ must have a decomposition

$$
M_{x}=S_{1} \oplus \ldots \oplus S_{r},
$$

where $S_{i}$ is a simple $B$-module of dimension $d_{i}$ and all components are distinct. That is, $n=\sum_{i} d_{i}$ and the embedding of $G L(\mathbf{e})=\mathbb{C}^{*} \times \ldots \times \mathbb{C}^{*}(r$ factors $)$ in $G L_{n}$ is given via

$$
\left(\lambda_{1}, \ldots, \lambda_{r}\right) \mapsto \operatorname{diag}(\underbrace{\lambda_{1}, \ldots, \lambda_{1}}_{d_{1}}, \ldots, \underbrace{\lambda_{r}, \ldots, \lambda_{r}}_{d_{r}}) .
$$

We want to describe the étale local structure of $A$ near $m$, that is, the $\operatorname{ring} A_{m}^{s h}=$ $A \otimes_{Z} Z_{m}^{s h}$. In order to do this, we have to compute the rings of invariants and concomitants of the local chart near the zero representation.

Proposition 6.4. Using the labeling of vertices and arrows in the chart $A_{k l m}$ given above, we have: 
1. The ring of polynomial invariants is equal to

$$
\mathbb{C}\left[R\left(A_{k l m}, \epsilon\right) / G L(\mathbf{e})\right]=\mathbb{C}[x, y] .
$$

2. The rings of $G L_{n}$-equivariant maps

$$
M_{n}\left(G L_{n} \times{ }^{G L(\mathbf{e})} \mathbb{C}\left[R\left(A_{k l m}, \epsilon\right]\right)^{G L_{n}}\right.
$$

is isomorphic to the subring of $M_{n}(\mathbb{C}[x, y])$ with block decomposition

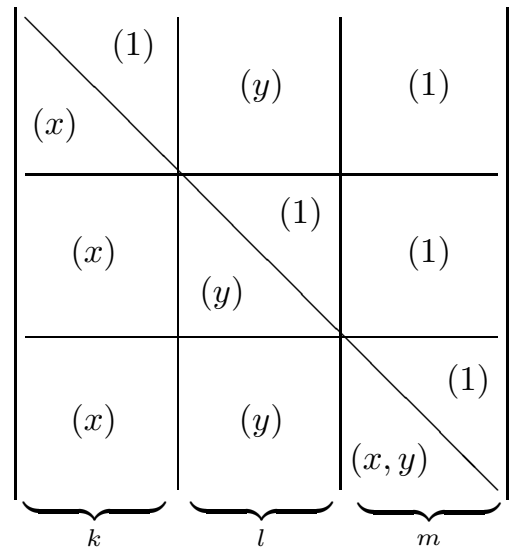

where at place $(i, j)$ (for every $1 \leq i, j \leq r$ ) there is a block of dimension $d_{i} \times d_{j}$ with entries the indicated ideal of $\mathbb{C}[x, y]$.

Proof. By basechange in the vertices we see that all non-zero maps in a minimal oriented cycle can be taken to be the identity map, except for one. If we define these remaining maps $x$ and $y$, then the traces along oriented cycles in the chart are of the form $x^{i} y^{j}$. The result about the equivariant maps follows from computing the $\mathbb{C}[x, y]$-module of paths from a vertex $(i)$ to vertex $(j)$ and applying the general results of the previous section.

Using the Luna slice theorem, we obtain the required étale local classification

Theorem 6.5. With notations as before, we have:

1. $Z_{m}^{s h} \simeq \mathbb{C}\{x, y\}$.

2. $A_{m}^{\text {sh }}$ is isomorphic to the subring of $M_{n}(\mathbb{C}\{x, y\})$ with the above block decomposition.

Definition 6.6. A $Z$-order $A$ in a central simple algebra $\Delta$ of dimension $n^{2}$ is said to be étale locally split in a maximal ideal $m$ of $Z$ iff $B_{m}^{s h}$ has ring of fractions $M_{n}\left(K \otimes_{Z} Z_{m}^{s h}\right)$.

From the étale local description of $Z$ and $B$ and étale descent we deduce

Proposition 6.7. If $A$ is a smooth order with two-dimensional center, then:

1. The center $Z$ is smooth.

2. The non-Azumaya locus of $A, \operatorname{ram}_{A}=Y_{A}-Y_{A z}$, consists at worst of isolated (possibly embedded) points and a reduced divisor whose worst singularities are normal crossings.

3. A is étale locally split at every point $m \in Y_{A}$. 
Proof. (1) and (3) are immediate from the foregoing theorem. For (2), we have to study the local charts in proper semi-simple representations of $R\left(A_{k l m}, \mathbf{e}\right)$.

Their decomposition into simple representations can be depicted by one of the following two situations:
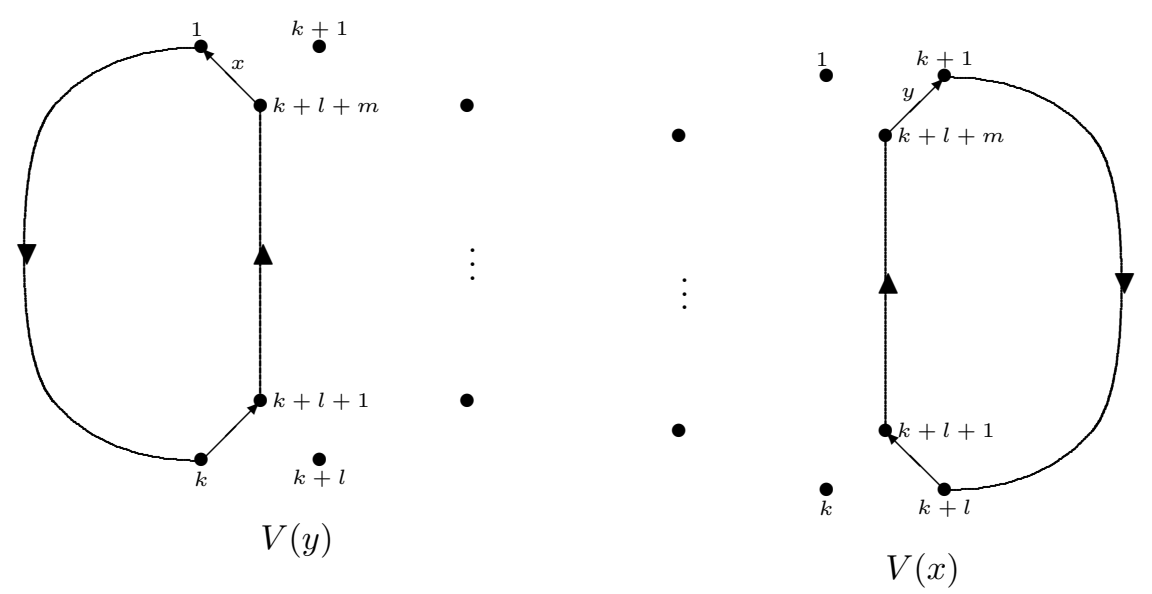

where the trace along the indicated oriented cycle is non-zero. By the general results of the foregoing section we can compute the local charts of $A$ near such a point. They are resp. of the following types:

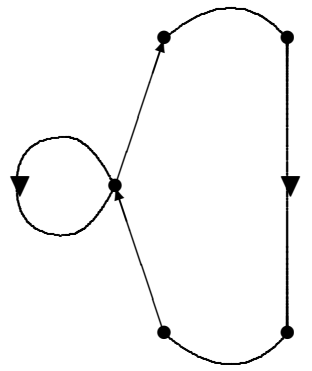

$A_{0 l 1}$

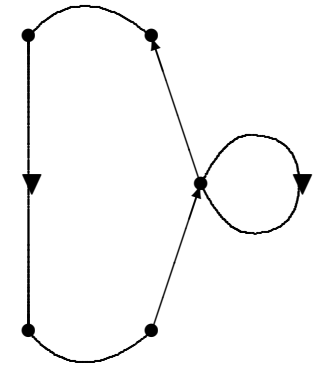

$A_{k 01}$

and we have the following local picture of the structure of $Y_{A}$ near $m$ : 


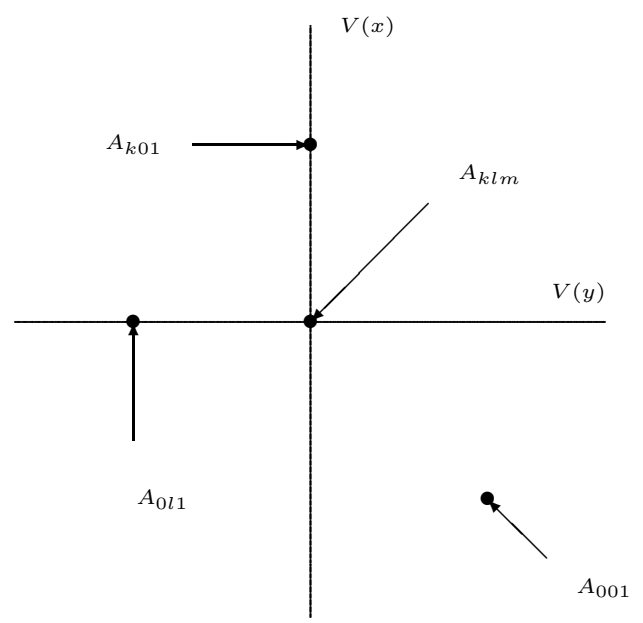

from which the statement follows (taking care of possible degenerate cases; for example, an isolated point occurs for local charts of type $A_{00 m}$ with $m \geq 2$ ).

We see that already in dimension two, smooth orders do not have to have finite global dimension.

Lemma 6.8. With notations as before, $A$ is a projective module over $Z$ if and only if all local charts are of type $A_{k l 1}$. In particular, if a local chart is of type $A_{k l m}$ with $m \geq 2$, then gldim $A=\infty$.

Proof. As the center is smooth, projectivity and reflexivity as $Z$-module are equivalent. Observe that $A_{m}^{s h}$ is reflexive only if no block of type $(x, y)$ occurs, that is, iff $m=1$. The last statement follows from the fact that an order of finite global dimension with smooth center has to be projective.

From dimension $d=3$ on some new phenomena occur. Rather than giving a full classification, we will give examples of the differences.

Lemma 6.9. In dimension $d \geq 3$ the center $Z$ of a smooth order $A$ in $C H_{n}$ no longer has to be smooth.

Proof. Consider the local chart of the form

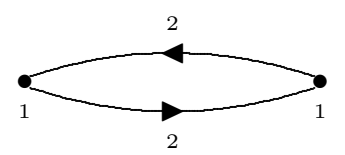

The center of the corresponding ring of equivariant maps is equal to

$$
\mathbb{C}[x, y, z, v] /(x y-z v)
$$

and hence is not smooth in the origin. In arbitrary dimension $d \geq 3$ this example survives by adding loops in one of the vertex spaces.

Lemma 6.10. In dimension $d \geq 3$ a smooth algebra $A$ does not have to be étale locally split everywhere. 
Proof. Consider the local chart

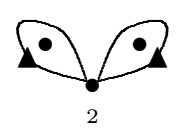

Then one verifies that the ring of equivariant maps is equal to

$$
\operatorname{Cliff}_{\mathbb{C}[x, y, z]}\left[\begin{array}{ll}
x & y \\
y & z
\end{array}\right],
$$

the Clifford algebra of a non-degenerate quadratic form. This algebra cannot be split by an étale extension at the origin.

\section{REFERENCES}

[1] J. Cuntz and D. Quillen, Cyclic homology and nonsingularity, Journal of the AMS 8 (1995), 373-442 MR 96e:19004

[2] J. Cuntz and D. Quillen, Algebra extensions and nonsingularity, Journal of the AMS 8 (1995), 251-289 MR 96c:19002

[3] B. Iversen, Generic local structure in commutative algebra, Lecture Notes in Math. 310 Springer-Verlag, Berlin, 1973. MR 50:13023

[4] H. Kraft, Geometrische Methoden in der Invariantentheorie, Aspecte der Mathematik D1 F. Vieweg, Braunschweig, 1984 MR 86j:14006

[5] L. Le Bruyn and C. Procesi, Etale local structure of matrix-invariants and concomitants, in Algebraic Groups, Utrecht 1986, Lecture Notes in Math. 1271, Springer-Verlag, Berlin, 1987, pp. 143-176 MR 89b:16042

[6] L. Le Bruyn and C. Procesi, Semi-simple representations of quivers, Trans. AMS 317 (1990) 585-598 MR 90e:16048

[7] D. Luna, Slices étales, Bull. Soc. Math. France, Mémoire 33 (1973) 81-105 MR 49:7269

[8] C. Procesi, A formal inverse to the Cayley-Hamilton theorem, J. of Algebra 107 (1987), 63-74 MR 88b:16033

[9] I. Reiner, Maximal orders, Academic Press (1975) MR 52:13910

[10] W. Schelter, Smooth algebras, J. of Algebra 103 (1986), 677-685 MR 88a:16034

[11] P. Slodowy, Der Scheibensatz für algebraische Transformationsgruppen, Algebraische Transformationsgruppen und Invariantentheorie, DMV Sem. 13, Birkhäuser, Basel, 1989, pp. 89113 MR 91m:14074

Departement Wiskunde, University of Antwerp (UIA) B.2610, Antwerp, Belgium

E-mail address: lebruyn@wins.uia.ac.be 